\title{
A STUDY OF SERUM BETA LIPOPROTEIN AND TOTAL CHO- LESTEROL VARIABILITY AND ITS RELATION TO AGE AND SERUM LEVEL IN ADULT HUMAN SUBJECTS ${ }^{1}$
}

\author{
By DONALD M. WATKIN, ELEANOR Y. LAWRY, GEORGE V. MANN, AND \\ MAX HALPERIN \\ (From the Section on Gerontology, National Heart Institute, National Institutes of Health, \\ P.H.S., D.H.E. and W., Bethesda, Md., and the Baltimore City Hospitals, Baltimore, \\ Md., and the Department of Nutrition, Harvard University School of Public \\ Health, Boston, Mass.)
}

(Submitted for publication November 20, 1953; accepted January 6, 1953)

This study is concerned with an evaluation of the nature and magnitude of the variability of the serum cholesterol and certain classes of serum lipoproteins in adult men. The immediate objectives of the experiments described were three: (1) to study the relation of age and mean serum level to the day-to-day variation in serum lipid levels, (2) to characterize the magnitude and origin of the variability encountered, and (3) to define the number of samples which must be analyzed to yield reliable values.

Despite the abundance of cholesterol in human blood and the many discussions about its significance, descriptions of "normal" levels have been diffuse and often inconsistent. There has been no agreement upon the extent of individual variability with time nor variability in different physiological states. The lack of uniformity in results can be attributed in part to the technical variability or nonspecificity of the difficult analytical methods. It may also reflect a lability of biological control.

The nature of variability in relation to serum and tissue cholesterol as well as to the other lipids measured by chemical methods has been discussed extensively by Bloor (1) and more specifically by Bang (2). The influence of meals and the existence of a diurnal variation of serum cholesterol have been reported by McClure and Huntsinger (3), Bruger and Somach (4), and Boyd (5) among others.

1 The laboratory analyses reported in this paper were performed in the Department of Nutrition at the Harvard University School of Public Health and were supported in part by grants in aid from the National Heart Institute, National Institutes of Health, Bethesda; the Nutrition Foundation, New York; the J. M. Kaplan Fund, New York; and the American Meat Institute, Chicago.
Turner and Steiner (6), using the method of Bloor, Pelkan, and Allen, have reported on variation in total serum cholesterol measured at weekly intervals in ten patients at four periods during 63 weeks of investigation. They noted that the maximum per cent deviation of any single total cholesterol determination from an individual's mean for all values ranged from four per cent (mean 276 mg. per cent) to 15 per cent (mean $280 \mathrm{mg}$. per cent). The maximum deviation was less than ten per cent in six cases (average of individual means $283 \mathrm{mg}$. per cent). Steiner and Domanski (7), using the same technique, later reported a mean standard deviation of $8.7 \mathrm{mg}$. per cent in 15 normals with a mean total cholesterol level of $254 \mathrm{mg}$. per cent and a mean standard deviation of $24.8 \mathrm{mg}$. per cent in 15 patients with coronary atherosclerosis with a mean level of $355 \mathrm{mg}$. per cent. Both groups were studied regularly over a two year period. Man and Gildea (8) studied ten young subjects for periods of three to 48 months and found that the range of total cholesterol for individuals varied from one per cent (mean $237 \mathrm{mg}$. per cent) to 31 per cent (mean $213 \mathrm{mg}$. per cent) of the mean. Man and Peters (9) reexamined 16 of the same subjects 10 to 20 years later and presented expanded data describing the variability of cholesterol within individuals on six subjects from the original group which revealed coefficients of variation ranging from 4.9 to 9.9 per cent. Sperry (10) studied 25 young subjects for periods of 28 months and found in 17 cases a variation from the mean of 6.2 per cent or less with a maximum variation of 12.3 per cent. He reported an average of the individual standard deviations of $12.1 \mathrm{mg}$. per cent. He concluded that an individual has a char- 
acteristic level from which large deviations do not occur.

Schube (11) studied the variations of blood cholesterol of ten male subjects 18 to 35 years of age whose levels were measured in duplicate at weekly intervals over a period of 16 weeks by a modification of the Myers-Wordell procedure applied to whole blood. He found the greatest week to week change in an individual to be $73 \mathrm{mg}$. per cent (mean $143 \mathrm{mg}$. per cent) and the maximum deviations from the means of individuals to range from 19 (mean $132 \mathrm{mg}$. per cent) to 47 (mean $143 \mathrm{mg}$. per cent) mg. per cent.

The individual variability of serum lipoproteins has not been described in detail. Gofman and his associates (12) refer to this with the statement that two measurements 2 to 90 days apart in approximately 65 individuals revealed "that on a constant diet, a single individual shows a reasonably stable level of concentration of such molecules, whether the concentration is low or high." They justify the adequacy of a single sample drawn at any time of day in this manner.

In another discussion, "standard errors" for the $45 \mathrm{mg}$. per cent level of Sf 12-20 molecules and for the $243 \mathrm{mg}$. per cent level of cholesterol were presented by the same workers (13). These values were respectively $\pm 10 \mathrm{mg}$. per cent and $\pm 22 \mathrm{mg}$. per cent for "biologic variation" and $\pm 14 \mathrm{mg}$. per cent and $\pm 26 \mathrm{mg}$. per cent for "over-all variation." They derived these values from estimates of "the standard deviation of distribution of levels" among 273 "normal" males and from "coefficients of reliability" based on two determinations in an undisclosed number of individuals whose blood was sampled under conditions and at intervals which were not described. They conclude that a single measurement will place the average individual within $\pm 14 \mathrm{mg}$. per cent for Sf 12-20 molecules and within $\pm 26 \mathrm{mg}$. per cent for cholesterol of the "true" levels two-thirds of the time. Gofman and his associates (14) subsequently presented values differing only slightly arrived at by a similar technique.

A detailed study of spontaneous and induced variation of serum cholesterol and certain classes of lipoproteins during short time intervals (hours) has been made (15). It was observed that spontaneous variability within an individual was in part dependent upon the level of the particular lipid. Variability at high lipid levels exceeded that of subjects with low levels. In certain individuals, a large biological variation (i.e., exceeding technical variations) was observed between successive bleedings.

For the present studies, a group of adult American males consuming a self-selected diet in habitual amounts and living in a free but medically observed environment to which the subjects were well adjusted was sought. The subjects studied have approached these requirements in most respects.

\section{EXPERIMENTAL PLAN}

Three successive studies have been carried out over a period of 13 months. The subjects were, for the most part, groups of adult and of elderly men who were for purposes of this investigation housed in the Hospital for Chronic Illness of the Baltimore City Hospitals. These men were indigent public wards and were not institutionalized primarily because of the presence of disease. In study I, a supplementary group of six adult males, all staff members of the Department of Nutrition at the Harvard University School of Public Health, were studied simultaneously in Boston.

Subjects were excluded who demonstrated prior to the start of the studies diabetes mellitus, definite hepatic insufficiency, the nephrotic syndrome, any known disease of lipid metabolism, hypo- or hyperthyroidism, known malignant disease, an acute infection within two weeks, severe anemia, marked emaciation, marked obesity, active syphilis, active tuberculosis, a surgical procedure within 60 days, hormone or radiation therapy within 60 days, or any anticoagulant therapy with heparin within 60 days. No effort was made to select or reject patients on the basis of atherosclerosis on the grounds that available techniques are inadequate to give more than a very gross estimate of the extent of atheromatous disease. With the exceptions noted in study I, blood was drawn from each subject twice weekly on Monday and Thursday for periods of ten weeks.

In Study I, laboratory measurements were not carried out in duplicate. Both the length of the study period and the frequency of blood samplings varied among the different individuals. In addition, 6 of the 22 subjects were at work in Boston, not institutionalized in Baltimore. Because of these variables, the data in study I will be considered separately. The second and third studies were planned to eliminate these imperfections and the data obtained have been pooled for the present evaluation. Studies II and III were carried out from March to August of 1952.

In the first study, approximately equal numbers of subjects in the age categories 26 to 41,47 to 58,63 to 76 , and 80 to 92 years were used. In the second and third studies, the age categories of subjects were 47 to 59,61 to 69 , 
TABLE I*

Composition and constancy of diet as presented and as actually consumed

\begin{tabular}{|c|c|c|c|c|}
\hline & Protein & Fat & Carbohydrate & Calories \\
\hline & $G m$. & $\mathrm{Gm}$. & $G m$ & \\
\hline $\begin{array}{l}\text { Menu analyses } \\
\text { Consumption measurements } \\
\text { (12-3 d. periods) }\end{array}$ & $\begin{array}{ll}82 & \pm 3 \\
60 & \pm 3\end{array}$ & $\begin{array}{l}95 \pm 2 \\
66 \\
\pm 5\end{array}$ & $\begin{array}{ll}262 & \pm 6 \\
217 & \pm 15\end{array}$ & $\begin{array}{l}2236 \pm 40 \\
1700 \pm 113\end{array}$ \\
\hline $\begin{array}{l}\text { Average S.E. of consumption } \\
\text { (within individuals) }\end{array}$ & $5.7 \pm 0.7$ & $6.3 \pm 0.8$ & $18.8 \pm 1.5$ & $76 \pm 17$ \\
\hline
\end{tabular}

* The top line shows mean values and standard errors for the components of 28 daily menus. The middle line shows the means and standard errors of the averages for daily consumption during each of twelve three-day periods. The bottom line shows the averages of twelve standard errors of the mean each of which was computed for one of twelve three-day periods.

73 to 79 , and 80 to 92 years. An attempt was made to include a range of Sf 12-20 lipoprotein values in each age category. In studies I and II a selection for lipoprotein level was attempted by using a single determination of total cholesterol as performed in the Baltimore City Hospitals clinical laboratory by the Bloor technique (modified) as the sole indicator. In study III, selection for lipoprotein level was based on a single preliminary Sf 12-20 measurement.

Diets. Except for the young adults included in study I, the subjects were confined throughout each study to the hospital wards. They were fed the standard diet provided by the hospital kitchen.

The composition of the diet as presented to the subjects was determined by calculation using food tables of 28 daily menus selected at random during the fivemonth period of studies II and III. Results of the analysis appear in Table I. Inspection of the standard errors indicates the diet to be remarkably constant from day to day.

During twelve three-day periods in nine subjects, the food actually consumed from the diet presented was recorded and its composition estimated using food tables. For each of the twelve three-day periods a mean daily consumption and the standard error of this mean were computed. The average of the mean intakes and the average of the standard errors for all twelve periods appear in Table I. These values indicate that the average subject did not consume all of the food presented him but that actual food consumption within individual subjects varied little from day to day.

Laboratory. The blood samples were drawn prior to breakfast and at least 14 hours after the last meal. The clotted blood was allowed to retract at room temperature for two and one-half hours. The clots were ringed with clean glass stirring rods and the tubes centrifuged for 15 minutes at $2000 \mathrm{rpm}$.

After the serum was separated, an aliquot of six $\mathrm{ml}$. was placed in a randomly numbered screw top vial, packed in an ice-filled Dewar flask, and immediately shipped to Boston by air for analysis. During studies II and III, the serum obtained was divided into two six ml. aliquots, each aliquot was given a randomly selected code number, and the individual aliquots sent to the Bos- ton Laboratory ostensibly as separate sera. The analytical results corresponding to each code number were returned to Baltimore for decoding. This technique permitted a truly unbiased estimate of technical error.

The total cholesterol content of serum was measured by the method of Abell, Levy, Brodie, and Kendall (16). Recrystallized cholesterol, with a melting point of 147 to $149^{\circ} \mathrm{C}$., was used as a standard reference material measured with the samples processed each day. The serum lipoproteins of the Sf 12-20, Sf 21-35 and Sf 35-100 classes were measured by the technique of Gofman and his associates (17). No feasible reference substance was at hand for standardization of this procedure. During the course of this work, duplicate aliquots of ten serum samples were exchanged between the four laboratories of the Cooperative Study of Lipoproteins and Atherosclerosis at approximately monthly intervals. These data revealed no major deviations of either the cholesterol or lipoprotein methods used in the present study. Instrument calibration with a standard reference cell further minimized the likelihood of the existence of a technical drift in the lipoprotein measurement during the study.

\section{ANALYSIS OF DATA}

After decoding, the data were tabulated and the mean level and standard deviation of the distribution calculated for each lipoprotein fraction and for the total cholesterol for each subject. The means $(\overline{\mathbf{x}})$ and standard deviations $\left(S_{\mathbf{T}}\right)$ were computed from single analyses for 22 subjects in study I and 13 subjects in study III and from the average values of duplicate pairs in 33 subjects on whom these were available in studies II and III. ${ }^{2}$

2 Treatment of missing data: In those few instances where one value of a duplicate pair was missing (laboratory accident, breakage in transit, etc.) the missing member was considered equal to the remaining member of the pair in computation of $S_{\mathbf{r}}$. Estimates of $S_{\mathbf{y}}$ were computed in these instances using $n-1$ rather than $n$ pairs, since no value for the difference between duplicates could be assumed in the absence of one member of a pair. 
Exclusion of Data: In the 35 subjects on whom only single measurements were made, means and standard deviations were computed using all available data. No datum was discarded even though it could reasonably be expected to be an outlier.

No lipoprotein data were excluded because of missing cholesterol data. Five lipoprotein values were excluded because of recorded technical errors in preparative centrifugation and photographic records of errors in ultracentrifugation. Since it was possible to exclude outliers among lipoprotein values on the basis of these records, it was not considered advisable to process further for outliers. In the case of cholesterol measurements no such permanent records were available, so that testing for outliers among values obtained in duplicate was done statistically using the extreme deviate test (18).

In this latter group a difference between duplicate cholesterol determinations which was demonstrated to be an outlier at the one per cent level of significance resulted in both members of that duplicate pair being discarded, even though one of the values may have agreed well with other data on the individual under consideration. There were 19 pairs of cholesterol observations so discarded out of a total of more than 600 . No other cholesterol data were discarded even in the absence of lipoprotein data for a given sample.

The variance among duplicates $\left(\mathrm{S}_{\mathrm{E}}{ }^{2}\right)$ was computed from duplicate measurements for each of these 33 subjects and was used for adjusting the individual's total variance. In this way an estimate of residual or what we shall call "biologic variation" $\left(\mathrm{S}_{\mathbf{B}}\right)$ was obtained. The computation for this adjustment was performed according to the formula:

$$
\mathrm{nS}_{\mathrm{B}}^{2}=\mathrm{nS}_{\mathrm{T}}{ }^{2}-1 / 2 \mathrm{nS}_{\mathrm{ER}}^{2}
$$

For the 35 subjects in studies I and III on whom measurements in duplicate were not available, a $\mathrm{S}_{\mathrm{E}}{ }^{2}$ used in correcting these individuals' total variance for technical error was arrived at by using the pooled variances among duplicates on those subjects having mean levels of the lipid fraction measured within ten per cent of the level to be corrected. To facilitate this correction, charts

TABLE II

Study I: Data for individuals and age groups

\begin{tabular}{|c|c|c|c|c|c|c|c|c|c|c|c|c|c|c|c|c|c|}
\hline \multirow{3}{*}{ Subject } & \multirow{3}{*}{$\begin{array}{c}\text { Age } \\
\text { (yrs.) }\end{array}$} & \multirow{3}{*}{$\begin{array}{c}\text { Weight } \\
(\boldsymbol{K} \boldsymbol{g} \cdot)\end{array}$} & \multirow{3}{*}{$\begin{array}{c}\text { Height } \\
\text { (cm.) }\end{array}$} & \multicolumn{10}{|c|}{ Beta lipoproteins } & \multirow{2}{*}{\multicolumn{4}{|c|}{$\frac{\text { Total cholesterol }}{\text { (mg. per cent) }}$}} \\
\hline & & & & \multirow{2}{*}{$\mathbf{n}$} & \multicolumn{3}{|c|}{$\begin{array}{c}S_{f 12-20} \\
\text { (mg. per cent) }\end{array}$} & \multicolumn{3}{|c|}{$\begin{array}{c}S_{t 21-35} \\
\text { (mg. per cent) }\end{array}$} & \multicolumn{3}{|c|}{$\begin{array}{c}S, 35-100 \\
\text { (mg. per cent) }\end{array}$} & & & & \\
\hline & & & & & $\bar{x}$ & $\mathbf{S}_{\mathbf{T}}$ & $\mathbf{S}_{\mathbf{B}}$ & $\overline{\mathbf{x}}$ & ST & $\mathbf{S B}_{\mathbf{B}}$ & $\bar{x}$ & $\mathbf{S}_{\mathbf{r}}$ & $\mathrm{S}_{\mathbf{B}}$ & $\mathbf{a}$ & $\bar{x}$ & $\mathbf{S}_{\mathbf{T}}$ & $\mathbf{S}_{\mathbf{B}}$ \\
\hline $\begin{array}{l}\text { J. Dg. } \\
\text { L. F. } \\
\text { T. V. } \\
\text { G. M. } \\
\text { D. H. } \\
\text { F. S. }\end{array}$ & $\begin{array}{l}26 \\
28 \\
31 \\
34 \\
37 \\
41\end{array}$ & $\begin{array}{l}72.7 \\
76.8 \\
70.4 \\
64.5 \\
56.8 \\
68.2\end{array}$ & $\begin{array}{l}163 \\
175 \\
173 \\
165 \\
172 \\
178\end{array}$ & $\begin{array}{r}8 \\
7 \\
10 \\
9 \\
8 \\
10\end{array}$ & $\begin{array}{l}28.00 \\
45.43 \\
19.90 \\
19.44 \\
52.50 \\
35.50\end{array}$ & $\begin{array}{l}6.87 \\
9.50 \\
6.23 \\
3.21 \\
6.16 \\
6.20\end{array}$ & $\begin{array}{l}5.59 \\
8.52 \\
4.97 \\
\overline{3.61} \\
4.70\end{array}$ & $\begin{array}{l}17.75 \\
32.57 \\
12.20 \\
10.33 \\
23.89 \\
18.10\end{array}$ & $\begin{array}{l}\mathbf{6 . 3 4} \\
\mathbf{7 . 7 6} \\
2.04 \\
2.83 \\
5.58 \\
5.34\end{array}$ & $\begin{array}{l}5.90 \\
6.93 \\
1.60 \\
4.92 \\
4.81\end{array}$ & $\begin{array}{l}30.12 \\
70.14 \\
22.20 \\
19.00 \\
30.11 \\
39.30\end{array}$ & $\begin{array}{l}12.04 \\
19.38 \\
11.08 \\
9.27 \\
12.39 \\
16.54\end{array}$ & $\begin{array}{r}10.77 \\
17.06 \\
9.80 \\
7.91 \\
11.16 \\
15.64\end{array}$ & $\begin{array}{r}8 \\
7 \\
10 \\
9 \\
9 \\
10\end{array}$ & $\begin{array}{l}191.1 \\
251.0 \\
219.0 \\
220.4 \\
263.6 \\
263.0\end{array}$ & $\begin{array}{r}9.98 \\
12.86 \\
15.94 \\
8.26 \\
15.73 \\
25.04\end{array}$ & $\overline{\overline{10.30}} \overline{\overline{1}}$ \\
\hline $\begin{array}{l}\overline{X^{*}} \\
S_{\bar{x}}+\end{array}$ & $\begin{array}{c}32.8 \\
2.30\end{array}$ & $\begin{array}{r}68.23 \\
2.84\end{array}$ & $\begin{array}{r}171.0 \\
2.38\end{array}$ & & $\begin{array}{r}33.46 \\
5.54\end{array}$ & $\begin{array}{l}6.36 \\
2.59\end{array}$ & $\begin{array}{l}4.57 \\
1.14\end{array}$ & $\begin{array}{r}19.14 \\
3.32\end{array}$ & $\begin{array}{l}4.98 \\
2.79\end{array}$ & $\begin{array}{l}4.03 \\
1.09\end{array}$ & $\begin{array}{r}35.14 \\
7.57\end{array}$ & $\begin{array}{r}13.45 \\
1.54\end{array}$ & $\begin{array}{r}12.06 \\
1.44\end{array}$ & & $\begin{array}{r}234.69 \\
11.90\end{array}$ & $\begin{array}{r}14.64 \\
2.43\end{array}$ & $\begin{array}{l}4.87 \\
3.27\end{array}$ \\
\hline $\begin{array}{l}\text { T. Bt. } \\
\text { G. S. } \\
\text { E. He. } \\
\text { F. P. } \\
\text { T. Q. }\end{array}$ & $\begin{array}{l}47 \\
48 \\
52 \\
58 \\
58\end{array}$ & $\begin{array}{l}68.7 \\
59.8 \\
54.5 \\
65.8 \\
52.7\end{array}$ & $\begin{array}{l}173 \\
170 \\
170 \\
170 \\
164\end{array}$ & $\begin{array}{r}7 \\
16 \\
17 \\
16 \\
14\end{array}$ & $\begin{array}{l}45.71 \\
27.38 \\
31.12 \\
19.81 \\
16.21\end{array}$ & $\begin{array}{r}11.57 \\
6.11 \\
7.66 \\
4.20 \\
3.19\end{array}$ & $\begin{array}{r}10.78 \\
4.71 \\
6.50 \\
1.89 \\
-\end{array}$ & $\begin{array}{r}23.29 \\
19.12 \\
19.41 \\
10.94 \\
8.57\end{array}$ & $\begin{array}{l}6.58 \\
4.46 \\
4.35 \\
2.79 \\
4.09\end{array}$ & $\begin{array}{l}\mathbf{6 . 0 3} \\
3.80 \\
3.67 \\
1.54 \\
\mathbf{3 . 5 1}\end{array}$ & \begin{tabular}{r|}
32.57 \\
25.69 \\
38.18 \\
8.19 \\
12.93
\end{tabular} & \begin{tabular}{r|}
18.70 \\
11.51 \\
14.38 \\
5.17 \\
8.18
\end{tabular} & \begin{tabular}{r|}
17.91 \\
10.28 \\
13.33 \\
1.81 \\
7.01
\end{tabular} & $\begin{array}{r}9 \\
15 \\
17 \\
16 \\
14\end{array}$ & $\begin{array}{l}160.8 \\
174.5 \\
263.4 \\
133.5 \\
289.1\end{array}$ & $\begin{array}{r}8.94 \\
11.91 \\
25.85 \\
19.61 \\
19.37\end{array}$ & $\begin{array}{r}\overline{1.46} \\
19.97 \\
15.65 \\
10.30\end{array}$ \\
\hline$\frac{\bar{x}}{s_{\bar{x}}}$ & $\begin{array}{c}52.6 \\
2.36\end{array}$ & $\begin{array}{r}60.30 \\
3.10\end{array}$ & $\begin{array}{r}169.4 \\
1.47\end{array}$ & & $\begin{array}{r}28.05 \\
5.15\end{array}$ & $\begin{array}{l}6.55 \\
4.66\end{array}$ & $\begin{array}{l}4.78 \\
1.87\end{array}$ & $\begin{array}{r}16.27 \\
2.78\end{array}$ & $\begin{array}{l}4.45 \\
1.93\end{array}$ & $\begin{array}{l}3.71 \\
0.71\end{array}$ & $\begin{array}{r}23.51 \\
5.70\end{array}$ & $\begin{array}{r}11.59 \\
2.36\end{array}$ & $\begin{array}{r}10.07 \\
2.74\end{array}$ & & $\begin{array}{r}204.26 \\
30.39\end{array}$ & $\begin{array}{r}17.14 \\
3.01\end{array}$ & $\begin{array}{l}9.48 \\
3.89\end{array}$ \\
\hline $\begin{array}{l}\text { W. Re. } \\
\text { B. S. } \\
\text { H. C. } \\
\text { P. K. } \\
\text { J. Me. } \\
\text { F. H. }\end{array}$ & $\begin{array}{l}63 \\
64 \\
65 \\
73 \\
73 \\
76\end{array}$ & $\begin{array}{l}\mathbf{6 2 . 5} \\
\mathbf{5 4 . 2} \\
\mathbf{5 9 . 7} \\
\mathbf{5 5 . 5} \\
\mathbf{5 7 . 1} \\
\mathbf{5 1 . 1}\end{array}$ & $\begin{array}{l}165 \\
159 \\
168 \\
162 \\
159 \\
168\end{array}$ & $\begin{array}{r}16 \\
17 \\
16 \\
17 \\
8 \\
17\end{array}$ & $\begin{array}{l}26.81 \\
18.12 \\
24.25 \\
36.06 \\
20.75 \\
28.65\end{array}$ & $\begin{array}{r}6.35 \\
4.96 \\
5.32 \\
10.30 \\
4.83 \\
5.52\end{array}$ & $\begin{array}{l}4.94 \\
3.31 \\
3.53 \\
9.47 \\
2.73 \\
3.82\end{array}$ & $\begin{array}{r}15.19 \\
8.65 \\
13.94 \\
18.41 \\
19.62 \\
13.50\end{array}$ & $\begin{array}{l}5.60 \\
2.85 \\
3.13 \\
5.75 \\
3.89 \\
4.23\end{array}$ & $\begin{array}{l}5.03 \\
1.93 \\
2.09 \\
5.25 \\
3.11 \\
3.53\end{array}$ & $\begin{array}{l}24.63 \\
13.71 \\
20.00 \\
27.88 \\
49.88 \\
20.88\end{array}$ & $\begin{array}{r}12.34 \\
5.84 \\
6.89 \\
7.27 \\
14.31 \\
10.25\end{array}$ & $\begin{array}{r}11.21 \\
3.27 \\
4.55 \\
5.11 \\
13.26 \\
8.85\end{array}$ & $\begin{array}{r}16 \\
17 \\
16 \\
16 \\
9 \\
17\end{array}$ & $\begin{array}{l}219.8 \\
186.1 \\
205.9 \\
164.8 \\
197.1 \\
171.9\end{array}$ & $\begin{array}{l}15.25 \\
14.18 \\
16.68 \\
12.56 \\
13.76 \\
30.70\end{array}$ & $\begin{array}{r}9.18 \\
7.27 \\
11.41 \\
4.24 \\
6.42 \\
28.34\end{array}$ \\
\hline$\frac{\bar{x}}{S_{\bar{x}}}$ & $\begin{array}{c}69.0 \\
2.30\end{array}$ & $\begin{array}{r}56.68 \\
1.65\end{array}$ & $\begin{array}{r}163.5 \\
1.69\end{array}$ & & $\begin{array}{r}25.77 \\
2.59\end{array}$ & $\begin{array}{l}6.21 \\
2.68\end{array}$ & $\begin{array}{l}4.63 \\
1.01\end{array}$ & $\begin{array}{r}14.86 \\
1.60\end{array}$ & $\begin{array}{l}4.24 \\
1.57\end{array}$ & $\begin{array}{l}3.49 \\
0.58\end{array}$ & $\begin{array}{r}26.16 \\
5.13\end{array}$ & $\begin{array}{l}9.40 \\
1.41\end{array}$ & $\begin{array}{l}7.71 \\
1.64\end{array}$ & & $\begin{array}{r}190.92 \\
8.50\end{array}$ & $\begin{array}{r}17.19 \\
2.76\end{array}$ & $\begin{array}{r}11.14 \\
3.58\end{array}$ \\
\hline $\begin{array}{l}\text { F. B. } \\
\text { C. H. } \\
\text { B. M. } \\
\text { J. Mi. } \\
\text { J. W. }\end{array}$ & $\begin{array}{l}80 \\
85 \\
86 \\
91 \\
92\end{array}$ & $\begin{array}{l}\mathbf{5 9 . 1} \\
\mathbf{5 3 . 2} \\
\mathbf{5 2 . 7} \\
\mathbf{5 1 . 8} \\
\mathbf{5 6 . 8}\end{array}$ & $\begin{array}{l}165 \\
163 \\
155 \\
163 \\
156\end{array}$ & $\begin{array}{r}17 \\
16 \\
6 \\
16 \\
16\end{array}$ & $\begin{array}{l}37.47 \\
36.81 \\
83.83 \\
26.75 \\
37.50\end{array}$ & $\begin{array}{r}9.15 \\
8.50 \\
14.41 \\
5.94 \\
7.52\end{array}$ & \begin{tabular}{r|}
8.20 \\
7.47 \\
12.48 \\
4.40 \\
6.33
\end{tabular} & $\begin{array}{l}18.76 \\
26.31 \\
28.00 \\
10.31 \\
16.44\end{array}$ & $\begin{array}{l}4.59 \\
7.25 \\
5.29 \\
3.54 \\
4.93\end{array}$ & $\begin{array}{l}3.95 \\
6.76 \\
4.59 \\
2.66 \\
4.34\end{array}$ & $\begin{array}{l}68.88 \\
65.06 \\
55.67 \\
15.69 \\
19.19\end{array}$ & $\begin{array}{r}16.31 \\
29.15 \\
13.75 \\
8.93 \\
10.94\end{array}$ & $\begin{array}{r}14.41 \\
28.13 \\
11.93 \\
7.51 \\
9.81\end{array}$ & $\begin{array}{r}16 \\
17 \\
8 \\
17 \\
16\end{array}$ & $\begin{array}{l}232.9 \\
246.8 \\
237.9 \\
183.8 \\
196.8\end{array}$ & $\begin{array}{l}14.82 \\
22.94 \\
12.14 \\
12.14 \\
16.11\end{array}$ & $\frac{1 \overline{16.04}}{\overline{\overline{0.56}}}$ \\
\hline$\frac{\overline{\mathbf{X}}}{\mathbf{s}_{\bar{x}}}$ & $\begin{array}{l}86.8 \\
2.18\end{array}$ & $\begin{array}{r}54.72 \\
1.39\end{array}$ & $\begin{array}{r}160.4 \\
2.04\end{array}$ & & $\begin{array}{l}44.47 \\
10.05\end{array}$ & $\begin{array}{l}9.10 \\
4.53\end{array}$ & $\begin{array}{l}7.78 \\
1.34\end{array}$ & $\begin{array}{r}19.96 \\
3.26\end{array}$ & $\begin{array}{l}5.12 \\
1.92\end{array}$ & $\begin{array}{l}4.46 \\
0.66\end{array}$ & $\begin{array}{l}44.90 \\
11.43\end{array}$ & $\begin{array}{r}15.82 \\
3.56\end{array}$ & \begin{tabular}{r|}
14.36 \\
3.63
\end{tabular} & & $\begin{array}{r}219.61 \\
12.36\end{array}$ & $\begin{array}{r}15.63 \\
1.98\end{array}$ & $\begin{array}{l}\mathbf{5 . 3 2} \\
\mathbf{3 . 3 7}\end{array}$ \\
\hline
\end{tabular}

* Average of all values within an age group.

$\uparrow$ Standard error of the mean of all values within an age group. 
TABLE III

Studies II and III: Data for individuals and age groups

\begin{tabular}{|c|c|c|c|c|c|c|c|c|c|c|c|c|c|c|c|c|c|}
\hline \multirow{3}{*}{ Subject } & \multirow{3}{*}{$\begin{array}{c}\text { Age } \\
\text { (yrs.) }\end{array}$} & \multirow{3}{*}{$\begin{array}{l}\text { Weight } \\
\left(K_{\mathbf{g} .}\right)\end{array}$} & \multirow{3}{*}{$\underset{(\mathrm{cm} .)}{\text { Height }}$} & \multicolumn{10}{|c|}{ Beta lipoproteins } & \multicolumn{4}{|c|}{ Total cholesterol } \\
\hline & & & & & \multicolumn{3}{|c|}{$\begin{array}{c}\mathbf{S}_{f} 12-20 \\
\text { (mg. per cent) }\end{array}$} & \multicolumn{3}{|c|}{$\begin{array}{c}S_{f} 21-35 \\
\text { (mg. per cent) }\end{array}$} & \multicolumn{3}{|c|}{$\begin{array}{c}S_{f} 35-100 \\
\text { (mg. per cent })\end{array}$} & \multirow{2}{*}{$\mathbf{n}$} & \multicolumn{3}{|c|}{ (mg. per cent) } \\
\hline & & & & & $\bar{x}$ & ST & $S_{B}$ & $\bar{x}$ & ST & SB & $\overline{\mathbf{x}}$ & $\mathbf{S T}$ & $S_{B}$ & & $\bar{x}$ & $\mathbf{S r}$ & $\mathbf{S}_{\mathbf{B}}$ \\
\hline $\begin{array}{l}\text { A. Hr.* } \\
\text { V. E. } \\
\text { J.Ca. } \\
\text { W. C.* } \\
\text { B.C. } \\
\text { L. T. } \\
\text { E. Ha. }{ }^{*} \\
\text { J. Br.* } \\
\text { W. F. } \\
\text { J. Cz.* } \\
\text { L. P.* }\end{array}$ & $\begin{array}{l}47 \\
48 \\
50 \\
51 \\
51 \\
52 \\
53 \\
55 \\
55 \\
57 \\
59\end{array}$ & $\begin{array}{l}71.2 \\
68.7 \\
62.2 \\
69.0 \\
76.1 \\
62.4 \\
58.9 \\
70.3 \\
46.9 \\
66.7 \\
64.8\end{array}$ & $\begin{array}{l}176 \\
154 \\
171 \\
169 \\
162 \\
165 \\
171 \\
172 \\
175 \\
171 \\
165\end{array}$ & $\begin{array}{l}20 \\
17 \\
19 \\
19 \\
20 \\
20 \\
20 \\
19 \\
20 \\
20 \\
20\end{array}$ & \begin{tabular}{|l|}
21.02 \\
36.24 \\
25.50 \\
26.26 \\
12.10 \\
39.90 \\
58.22 \\
30.92 \\
26.40 \\
88.38 \\
66.62
\end{tabular} & $\begin{array}{r}5.86 \\
5.39 \\
3.62 \\
5.51 \\
4.13 \\
8.11 \\
8.49 \\
6.65 \\
5.84 \\
14.82 \\
6.52\end{array}$ & \begin{tabular}{|r|}
4.99 \\
3.40 \\
4.26 \\
1.50 \\
6.96 \\
6.82 \\
6.29 \\
4.17 \\
13.87 \\
4.25 \\
\end{tabular} & \begin{tabular}{|l|}
12.52 \\
16.35 \\
13.00 \\
15.90 \\
2.60 \\
18.55 \\
37.20 \\
17.53 \\
11.65 \\
38.98 \\
42.00
\end{tabular} & $\begin{array}{l}3.66 \\
4.26 \\
3.54 \\
3.36 \\
1.73 \\
3.83 \\
6.39 \\
6.52 \\
3.31 \\
7.98 \\
7.97\end{array}$ & $\begin{array}{l}3.40 \\
3.51 \\
2.61 \\
2.45 \\
2.99 \\
5.83 \\
6.30 \\
2.29 \\
7.48 \\
7.44\end{array}$ & $\begin{array}{l}37.85 \\
22.41 \\
24.84 \\
28.74 \\
3.00 \\
28.40 \\
66.05 \\
23.68 \\
12.45 \\
55.22 \\
51.42\end{array}$ & \begin{tabular}{|l|}
12.83 \\
10.31 \\
8.42 \\
7.56 \\
3.11 \\
6.89 \\
16.86 \\
10.32 \\
4.82 \\
13.08 \\
15.04
\end{tabular} & \begin{tabular}{|r|}
12.11 \\
8.82 \\
6.52 \\
5.41 \\
4.40 \\
15.86 \\
9.63 \\
11.80 \\
14.06
\end{tabular} & $\begin{array}{l}18 \\
19 \\
19 \\
19 \\
20 \\
20 \\
19 \\
17 \\
20 \\
20 \\
20\end{array}$ & $\begin{array}{l}202.7 \\
2339.1 \\
210.3 \\
205.8 \\
167.0 \\
259.8 \\
242.5 \\
160.0 \\
177.2 \\
271.2 \\
159.1\end{array}$ & \begin{tabular}{|l|}
11.28 \\
13.67 \\
17.46 \\
16.94 \\
31.65 \\
17.67 \\
15.41 \\
10.59 \\
31.00 \\
33.41 \\
10.93
\end{tabular} & $\begin{array}{r}9.45 \\
12.19 \\
14.14 \\
29.24 \\
5.36 \\
11.99 \\
7.27 \\
28.37 \\
27.76 \\
7.83\end{array}$ \\
\hline$\frac{\overline{\bar{x}} t}{\mathrm{~s}_{\mathrm{x}} \ddagger}$ & $\begin{array}{r}52.5 \\
1.1\end{array}$ & $\begin{array}{r}65.2 \\
2.2\end{array}$ & $\begin{array}{r}168.4 \\
1.9\end{array}$ & & $\begin{array}{r}39.23 \\
6.87\end{array}$ & $\begin{array}{l}6.81 \\
0.91\end{array}$ & $\begin{array}{l}5.14 \\
1.08\end{array}$ & $\begin{array}{r}20.57 \\
3.88\end{array}$ & $\begin{array}{l}4.78 \\
0.63\end{array}$ & $\begin{array}{l}4.03 \\
0.72\end{array}$ & $\begin{array}{r}32.82 \\
5.62\end{array}$ & $\begin{array}{l}9.93 \\
1.29\end{array}$ & $\begin{array}{l}8.06 \\
1.60\end{array}$ & & $\begin{array}{r}208.61 \\
12.13\end{array}$ & $\begin{array}{r}19.09 \\
2.62\end{array}$ & $\begin{array}{r}13.96 \\
3.03\end{array}$ \\
\hline $\begin{array}{l}\text { C. G.* } \\
\text { W. A.* } \\
\text { J. Mk.** } \\
\text { R. B.* } \\
\text { J. R.* } \\
\text { J.P.** } \\
\text { H. W. W. } \\
\text { L. Bk.* } \\
\text { P. D.* } \\
\text { W. L..* } \\
\text { W. T.* } \\
\text { T. R. }\end{array}$ & $\begin{array}{l}61 \\
63 \\
63 \\
64 \\
64 \\
65 \\
66 \\
67 \\
67 \\
68 \\
68 \\
69\end{array}$ & $\begin{array}{l}62.0 \\
60.3 \\
91.5 \\
62.6 \\
69.0 \\
57.7 \\
50.0 \\
47.0 \\
61.8 \\
56.1 \\
73.1 \\
53.4\end{array}$ & $\begin{array}{l}173 \\
164 \\
171 \\
168 \\
166 \\
175 \\
166 \\
170 \\
164 \\
167 \\
171 \\
173\end{array}$ & $\begin{array}{l}20 \\
20 \\
20 \\
20 \\
20 \\
20 \\
20 \\
19 \\
20 \\
20 \\
19 \\
20\end{array}$ & \begin{tabular}{|l|}
20.22 \\
26.92 \\
49.90 \\
54.90 \\
66.42 \\
50.25 \\
33.25 \\
33.68 \\
41.15 \\
54.48 \\
52.55 \\
30.55
\end{tabular} & $\begin{array}{r}3.68 \\
3.88 \\
8.56 \\
6.03 \\
10.49 \\
4.82 \\
6.43 \\
9.09 \\
8.95 \\
8.36 \\
6.70 \\
6.69\end{array}$ & $\begin{array}{l}2.67 \\
3.10 \\
7.41 \\
5.11 \\
9.75 \\
4.06 \\
4.90 \\
8.89 \\
8.40 \\
7.59 \\
5.25 \\
5.24\end{array}$ & \begin{tabular}{|l|}
10.98 \\
17.40 \\
22.10 \\
25.02 \\
15.68 \\
18.68 \\
11.75 \\
19.16 \\
22.05 \\
11.28 \\
25.55 \\
17.75
\end{tabular} & $\begin{array}{l}2.86 \\
3.08 \\
4.54 \\
4.27 \\
4.39 \\
4.75 \\
3.43 \\
4.54 \\
7.35 \\
3.27 \\
7.85 \\
4.92\end{array}$ & \begin{tabular}{|l|}
2.59 \\
2.21 \\
4.14 \\
3.60 \\
3.97 \\
4.47 \\
2.46 \\
4.16 \\
7.25 \\
2.92 \\
7.55 \\
4.30
\end{tabular} & $\begin{array}{l}18.98 \\
43.70 \\
63.75 \\
32.72 \\
19.28 \\
23.50 \\
24.50 \\
41.29 \\
26.30 \\
14.70 \\
34.60 \\
26.85\end{array}$ & $\begin{array}{r}8.56 \\
10.03 \\
14.99 \\
7.79 \\
5.75 \\
5.63 \\
8.11 \\
11.16 \\
9.12 \\
4.36 \\
15.38 \\
7.94\end{array}$ & $\begin{array}{r}7.82 \\
9.07 \\
14.08 \\
6.87 \\
4.64 \\
4.95 \\
6.14 \\
10.49 \\
8.81 \\
1.58 \\
14.85 \\
5.90\end{array}$ & $\begin{array}{l}19 \\
20 \\
18 \\
19 \\
20 \\
19 \\
20 \\
19 \\
19 \\
20 \\
17 \\
20\end{array}$ & $\begin{array}{l}177.2 \\
211.0 \\
195.2 \\
144.2 \\
204.8 \\
202.0 \\
205.0 \\
231.7 \\
202.6 \\
228.0 \\
259.1 \\
148.8\end{array}$ & $\begin{array}{l}32.15 \\
16.74 \\
20.16 \\
14.02 \\
13.44 \\
11.89 \\
22.29 \\
25.30 \\
15.54 \\
12.50 \\
31.09 \\
29.83\end{array}$ & $\begin{array}{r}30.41 \\
13.99 \\
19.45 \\
13.03 \\
5.41 \\
9.07 \\
18.46 \\
22.88 \\
14.76 \\
8.03 \\
28.78 \\
27.25\end{array}$ \\
\hline$\frac{\bar{X}}{S_{\bar{x}}}$ & $\begin{array}{r}65.4 \\
0.7\end{array}$ & $\begin{array}{r}62.1 \\
3.4\end{array}$ & $\begin{array}{r}168.9 \\
1.1\end{array}$ & & $\begin{array}{r}42.86 \\
4.02\end{array}$ & $\begin{array}{l}6.97 \\
0.62\end{array}$ & $\begin{array}{l}6.03 \\
0.67\end{array}$ & $\begin{array}{r}18.12 \\
1.46\end{array}$ & $\begin{array}{l}4.60 \\
0.45\end{array}$ & $\begin{array}{l}4.14 \\
0.49\end{array}$ & $\begin{array}{r}30.85 \\
3.93\end{array}$ & $\begin{array}{l}9.07 \\
0.99\end{array}$ & $\begin{array}{l}7.93 \\
1.11\end{array}$ & & $\begin{array}{r}200.80 \\
9.43\end{array}$ & $\begin{array}{r}20.41 \\
2.18\end{array}$ & $\begin{array}{r}17.60 \\
2.42\end{array}$ \\
\hline $\begin{array}{l}\text { H. K.* } \\
\text { J. Ba.* } \\
\text { A. Hn.** } \\
\text { J. By.* } \\
\text { A. K.: } \\
\text { P. B.* } \\
\text { J.S.* } \\
\text { A. A.* } \\
\text { J. Fr.* } \\
\text { J.Co. Co. } \\
\text { W. D.* } \\
\text { J. Dk.* }\end{array}$ & $\begin{array}{l}72 \\
73 \\
73 \\
73 \\
74 \\
75 \\
75 \\
77 \\
77 \\
78 \\
78 \\
79\end{array}$ & $\begin{array}{l}49.3 \\
65.7 \\
74.4 \\
61.2 \\
65.2 \\
73.1 \\
49.9 \\
58.6 \\
45.5 \\
61.9 \\
81.5 \\
65.7\end{array}$ & $\begin{array}{l}168 \\
166 \\
164 \\
173 \\
165 \\
158 \\
161 \\
168 \\
161 \\
170 \\
166 \\
163\end{array}$ & $\begin{array}{l}20 \\
20 \\
20 \\
20 \\
20 \\
20 \\
20 \\
14 \\
20 \\
19 \\
20 \\
20\end{array}$ & \begin{tabular}{|l|}
$\mathbf{5 0 . 1 5}$ \\
18.45 \\
33.08 \\
23.52 \\
23.30 \\
48.30 \\
57.62 \\
48.75 \\
35.75 \\
27.16 \\
75.82 \\
45.90
\end{tabular} & $\begin{array}{r}6.51 \\
3.06 \\
9.05 \\
4.90 \\
4.45 \\
7.26 \\
10.84 \\
6.46 \\
6.16 \\
4.98 \\
14.61 \\
5.54\end{array}$ & \begin{tabular}{|r|}
5.74 \\
0.23 \\
8.40 \\
3.80 \\
1.75 \\
6.82 \\
10.18 \\
5.82 \\
4.95 \\
2.83 \\
13.15 \\
5.03
\end{tabular} & \begin{tabular}{|l|}
17.98 \\
12.68 \\
25.70 \\
14.48 \\
16.45 \\
20.82 \\
21.32 \\
21.43 \\
14.80 \\
13.79 \\
44.55 \\
39.15
\end{tabular} & $\begin{array}{l}3.82 \\
2.39 \\
5.60 \\
4.29 \\
2.19 \\
4.14 \\
3.25 \\
3.21 \\
3.61 \\
2.82 \\
5.58 \\
8.98\end{array}$ & \begin{tabular}{|l}
3.51 \\
1.93 \\
5.23 \\
4.02 \\
3.73 \\
2.37 \\
2.91 \\
2.93 \\
1.49 \\
3.97 \\
8.68
\end{tabular} & $\begin{array}{r}23.80 \\
19.70 \\
65.22 \\
18.35 \\
40.05 \\
52.82 \\
36.08 \\
34.07 \\
17.00 \\
20.95 \\
90.92 \\
110.55\end{array}$ & $\begin{array}{r}8.08 \\
5.49 \\
17.02 \\
6.37 \\
4.77 \\
15.68 \\
5.46 \\
6.65 \\
7.22 \\
5.54 \\
18.26 \\
31.36\end{array}$ & $\begin{array}{r}7.48 \\
4.34 \\
16.00 \\
5.70 \\
15.26 \\
15.36 \\
5.88 \\
6.09 \\
1.57 \\
15.99 \\
30.86\end{array}$ & $\begin{array}{l}20 \\
20 \\
19 \\
20 \\
20 \\
19 \\
20 \\
14 \\
19 \\
20 \\
20 \\
19\end{array}$ & \begin{tabular}{|l}
204.8 \\
170.2 \\
197.6 \\
167.7 \\
204.7 \\
182.6 \\
323.3 \\
275.1 \\
153.5 \\
205.8 \\
153.7 \\
182.7
\end{tabular} & \begin{tabular}{|l|}
16.26 \\
22.67 \\
25.50 \\
18.16 \\
12.40 \\
16.31 \\
22.31 \\
18.66 \\
18.15 \\
12.49 \\
15.90 \\
18.58
\end{tabular} & $\begin{array}{r}14.20 \\
16.47 \\
21.21 \\
15.75 \\
15.46 \\
8.56 \\
13.03 \\
15.49 \\
13.53 \\
17.40\end{array}$ \\
\hline$\frac{\bar{x}}{s_{\bar{x}}}$ & $\begin{array}{r}75.3 \\
0.7\end{array}$ & $\begin{array}{r}62.7 \\
2.2\end{array}$ & $\begin{array}{r}165.2 \\
1.2\end{array}$ & & $\begin{array}{r}40.65 \\
4.86\end{array}$ & $\begin{array}{l}6.98 \\
0.92\end{array}$ & $\begin{array}{l}5.72 \\
1.04\end{array}$ & $\begin{array}{r}21.93 \\
2.92\end{array}$ & $\begin{array}{l}4.16 \\
0.54\end{array}$ & $\begin{array}{l}3.40 \\
0.62\end{array}$ & $\begin{array}{r}44.13 \\
8.81\end{array}$ & $\begin{array}{r}10.99 \\
2.33\end{array}$ & $\begin{array}{l}9.38 \\
2.51\end{array}$ & & $\begin{array}{r}201.81 \\
14.49\end{array}$ & $\begin{array}{r}18.12 \\
1.13\end{array}$ & $\begin{array}{r}12.59 \\
1.90\end{array}$ \\
\hline $\begin{array}{l}\text { H. B.* } \\
\text { R. P.* } \\
\text { L. S.* } \\
\text { W. Ra.** } \\
\text { J. T. T. } \\
\text { J.G.* } \\
\text { M. C. } \\
\text { J.'Fa. } \\
\text { J. Mc. } \\
\text { G. A.* } \\
\text { L. Ba.* }\end{array}$ & $\begin{array}{l}80 \\
80 \\
81 \\
81 \\
81 \\
82 \\
85 \\
85 \\
85 \\
87 \\
92\end{array}$ & $\begin{array}{l}73.2 \\
43.1 \\
75.9 \\
83.3 \\
62.7 \\
66.4 \\
58.7 \\
55.8 \\
53.3 \\
73.6 \\
53.0\end{array}$ & $\begin{array}{l}171 \\
161 \\
169 \\
170 \\
172 \\
168 \\
159 \\
166 \\
162 \\
159 \\
159\end{array}$ & $\begin{array}{l}20 \\
20 \\
20 \\
20 \\
20 \\
20 \\
20 \\
19 \\
19 \\
20 \\
20\end{array}$ & \begin{tabular}{|l|}
70.08 \\
15.58 \\
50.25 \\
20.18 \\
39.20 \\
27.58 \\
35.40 \\
25.53 \\
48.95 \\
38.68 \\
45.98
\end{tabular} & $\begin{array}{l}7.90 \\
3.05 \\
5.25 \\
4.05 \\
7.72 \\
4.38 \\
6.34 \\
4.71 \\
8.84 \\
5.09 \\
8.03\end{array}$ & $\begin{array}{l}6.81 \\
1.93 \\
4.07 \\
3.09 \\
6.50 \\
3.17 \\
4.78 \\
2.32 \\
7.72 \\
3.96 \\
7.55\end{array}$ & \begin{tabular}{|l|}
27.05 \\
9.72 \\
32.25 \\
10.72 \\
15.10 \\
16.90 \\
24.45 \\
14.26 \\
16.58 \\
17.82 \\
32.38
\end{tabular} & $\begin{array}{l}5.05 \\
3.01 \\
5.33 \\
2.01 \\
3.60 \\
3.71 \\
3.19 \\
4.77 \\
3.39 \\
2.16 \\
6.11\end{array}$ & $\begin{array}{l}4.68 \\
2.60 \\
4.54 \\
1.31 \\
2.69 \\
3.55 \\
1.69 \\
4.13 \\
2.39 \\
0.89 \\
5.72\end{array}$ & $\begin{array}{r}49.00 \\
15.80 \\
119.05 \\
24.02 \\
23.20 \\
27.02 \\
40.90 \\
35.47 \\
25.05 \\
27.15 \\
48.85\end{array}$ & $\begin{array}{r}9.13 \\
6.95 \\
22.12 \\
5.87 \\
8.22 \\
12.05 \\
6.77 \\
14.29 \\
8.00 \\
5.56 \\
14.20\end{array}$ & \begin{tabular}{|r}
8.36 \\
6.05 \\
20.40 \\
4.81 \\
6.28 \\
11.18 \\
3.90 \\
13.17 \\
5.98 \\
3.50 \\
13.65
\end{tabular} & $\begin{array}{l}20 \\
18 \\
20 \\
20 \\
20 \\
20 \\
20 \\
20 \\
20 \\
20 \\
19\end{array}$ & \begin{tabular}{|l}
234.7 \\
139.2 \\
192.9 \\
142.9 \\
218.2 \\
174.8 \\
140.7 \\
189.0 \\
210.0 \\
207.9 \\
155.1
\end{tabular} & \begin{tabular}{|r|}
7.44 \\
11.62 \\
14.02 \\
11.92 \\
18.46 \\
21.90 \\
15.77 \\
18.30 \\
25.81 \\
11.89 \\
11.87
\end{tabular} & $\begin{array}{r}\overline{9.70} \\
11.19 \\
10.68 \\
13.60 \\
19.31 \\
10.08 \\
13.38 \\
22.58 \\
14.06 \\
10.61\end{array}$ \\
\hline 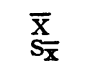 & $\begin{array}{r}83.5 \\
1.1\end{array}$ & $\begin{array}{r}63.5 \\
2.7\end{array}$ & $\begin{array}{r}165.1 \\
1.0\end{array}$ & & $\begin{array}{r}37.95 \\
4.72\end{array}$ & $\begin{array}{l}5.94 \\
0.58\end{array}$ & $\begin{array}{l}4.72 \\
0.63\end{array}$ & $\begin{array}{r}19.75 \\
2.42\end{array}$ & $\begin{array}{l}3.85 \\
0.40\end{array}$ & $\begin{array}{l}3.11 \\
0.46\end{array}$ & $\begin{array}{r}39.59 \\
8.58\end{array}$ & $\begin{array}{r}10.29 \\
1.51\end{array}$ & $\begin{array}{l}8.84 \\
1.58\end{array}$ & & $\begin{array}{r}182.31 \\
10.25\end{array}$ & $\begin{array}{r}15.82 \\
1.58\end{array}$ & $\begin{array}{r}12.29 \\
1.73\end{array}$ \\
\hline
\end{tabular}

* Values derived from duplicate determinations.

t Average of all values within an age group.

$\ddagger$ Standard error of the mean of all values within an age group.

relating the variance among duplicates to mean levels for each of the four lipid components were constructed. The correction was then performed in each of these 35 subjects according to the formula:

$$
\mathrm{nS}_{\mathrm{B}}{ }^{2}=\mathrm{nS}_{\mathrm{T}}{ }^{2}-\mathrm{nS}_{\mathrm{x}^{2}}
$$

Regression equations were computed relating the standard error among duplicate analyses $\left(\mathrm{S}_{\mathbf{E}}\right)$ to mean level, over-all variability $\left(S_{T}\right)$ to mean level, and biological variation $\left(S_{B}\right)$ to mean level. Correlation coefficients were computed relating mean cholesterol levels to mean levels of Sf 12-20, Sf 21-35, Sf 35-100 molecules and to the sums of these mean levels (Sf 12-100).

The data obtained during study I appear in Table II. The mean values for individuals as well 
as for groups of individuals for the age categories described above are shown along with measures of the variability including the biological variability $\left(S_{B}\right)$ computed according to the procedures described. Similar data for studies II and III are shown in Table III.

Since subjects were selected for study to provide among other things a wide range of lipoprotein levels in each age group, sampling was not strictly random. Thus in order properly to compare the $S_{\mathbf{T}}$ (and $S_{\mathbf{B}}$ ) among age groups some sort of adjustment for lack of randomness in sampling levels was necessary. This adjustment was made for studies II and III combined by the statistical technique known as the analysis of covariance. This involves verifying that the regressions of $S_{\mathbf{T}}$ (and $S_{B}$ ) on level have the same slope in each age group; if such is the case, it is then possible to make a regression adjustment for each value of $S_{T}\left(\right.$ and $S_{B}$ ) before testing age group averages for homogeneity. The results of the preliminary analysis implied that all within-age regressions for studies II and III had substantially the same slope.

The analysis of covariance was not performed for study I because of the various defects of that study previously described. Consequently the regression equations and statistical tests for study I should be viewed with some reservations. It is, however, interesting to note that at least in terms of absolute differences between pairs of slopes and intercepts, the two sets of regression equations are much the same.

Analysis of adjusted age group differences showed a statistically significant agewise difference in variability $(P=.05)$ only in the case of the Sf 21-35 fraction. However, in this last case,

TABLE IV

\section{Regression analysis of $S_{T}$ on $*$ for various lipoproteins and total cholesterol}

\begin{tabular}{cccc}
\hline \hline Study & $\mathrm{n}$ & Lipid & Regression $^{*}$ \\
\hline I & 22 & $\mathrm{~S}_{\mathrm{f}} 12-20$ & $\mathrm{~S}_{\mathrm{T}}=2.19+.147 \overline{\mathrm{x}}$ \\
& & $\mathrm{S}_{\mathrm{f}} 21-35$ & $\mathrm{~S}_{\mathrm{T}}=1.52+.181 \overline{\mathrm{x}}$ \\
& & $\mathrm{S}_{\mathrm{f}} 35-100$ & $\mathrm{~S}_{\mathrm{T}}=5.21+.225 \overline{\mathrm{x}}$ \\
& & Total Cholesterol & Not Significant \\
II \& III & 46 & $\mathrm{~S}_{\mathrm{f}} 12-20$ & $\mathrm{~S}_{\mathrm{T}}=2.00+.116 \overline{\mathrm{x}}$ \\
& & $\mathrm{S}_{\mathrm{f}} 21-35$ & $\mathrm{~S}_{\mathrm{T}}=1.48+.142 \mathrm{x}$ \\
& & $\mathrm{S}_{\mathrm{f}} 35-100$ & $\mathrm{~S}_{\mathrm{r}}=2.86+.196 \overline{\mathrm{x}}$ \\
& & Total Cholesterol & Not Significant \\
& & & \\
\hline
\end{tabular}

* Slopes significant, $\mathrm{p}<.001$.
TABLE V

Regression analysis of $S_{B}$ on $x$ for various lipoproteins and total cholesterol

\begin{tabular}{cccc}
\hline \hline Study & $\mathrm{n}$ & Lipid & Regression* $^{*}$ \\
\hline I & 22 & $\mathrm{~S}_{\mathrm{f}} 12-20$ & $\mathrm{~S}_{\mathrm{B}}=0.24+.157 \overline{\mathrm{x}}$ \\
& & $\mathrm{S}_{\mathrm{f}} 21-35$ & $\mathrm{~S}_{\mathrm{B}}=0.36+.202 \overline{\mathrm{x}}$ \\
& & $\mathrm{S}_{\mathrm{f}} 35-100$ & $\mathrm{~S}_{\mathrm{B}}=3.57+.228 \overline{\mathrm{x}}$ \\
& & Total Cholesterolt & \\
II \& III & 46 & $\mathrm{~S}_{\mathrm{f}} 12-20$ & $\mathrm{~S}_{\mathrm{B}}=0.26+.128 \mathrm{x}$ \\
& & $\mathrm{S}_{\mathrm{f}} 21-35$ & $\mathrm{~S}_{\mathrm{B}}=0.75+.146 \overline{\mathrm{x}}$ \\
& & $\mathrm{S}_{\mathrm{f}} 35-100$ & $\mathrm{~S}_{\mathrm{B}}=1.02+.204 \overline{\mathrm{x}}$ \\
& & Total Cholesterol & Not Significant \\
& & & \\
\hline
\end{tabular}

* Slopes significant, $\mathrm{p}<.001$.

† Since in 8 of the 22 cases the total variance for cholesterol was less than technical variance, computation of regression was not attempted.

since computation showed a difference of approximately one mg. per cent between the upper two and lower two age groups, there seemed, as a practical matter, little point in supplying separate regressions for each age class.

As a consequence of the analysis described above, it was considered justifiable to compute over-all regression lines of $S_{T}\left(\right.$ and $S_{B}$ ) on level for the various measures considered. For all of the lipoproteins, the regression slopes were significant at the .001 level. A significant regression of $S_{T}$ (and $S_{B}$ ) on mean level of total cholesterol could not be demonstrated. (Tables IV and V).

A highly significant regression was demonstrated between the standard errors among duplicates and the mean levels of both the lipoprotein fractions and the total cholesterol in the 33 subjects from studies II and III on whom duplicate analyses were available (Table VI). Regression equations indicate that the standard error among duplicates increases about $0.06 \mathrm{mg}$. per cent for each mg. per cent increment in mean level for all the lipoprotein fractions and about $0.08 \mathrm{mg}$. per cent for each $\mathrm{mg}$. per cent increment in total cholesterol.

The subjects were selected both for age and

TABLE VI

Regression analysis of $S_{E}$ on $x$ of lipoproteins and total cholesterol $(n=33)$

\begin{tabular}{cc}
\hline \hline Lipid & \multicolumn{2}{c}{ Regression* } \\
\hline$S_{\mathrm{f}} 12-20$ & $\mathrm{~S}_{\mathrm{E}}=2.35+.052 \overline{\mathrm{x}}$ \\
$\mathrm{S}_{\mathrm{f}} 21-35$ & $\mathrm{~S}_{\mathrm{E}}=1.23+.065 \overline{\mathrm{x}}$ \\
$\mathrm{S}_{\mathrm{f}} 35-100$ & $\mathrm{~S}_{\mathrm{E}}=3.45+.061 \overline{\mathrm{x}}$ \\
Cholesterol & $\mathrm{S}_{\mathrm{E}}=-2.81+.079 \overline{\mathrm{x}}$
\end{tabular}

* Slopes significant, $p<.001$. 
serum lipid levels according to the several procedures described above. It was observed that the single determination of serum cholesterol on each subject performed in the clinical laboratory of the Baltimore City Hospitals was significantly correlated with the mean total cholesterol level of that individual for the entire study but this single cholesterol measurement did not successfully predict the mean level of the lipoprotein classes. The initial Sf 12-20 measurement effectively predicted the mean level of Sf 12-20 and Sf 21-35 but not Sf 35-100.

An examination of the relations between mean levels of total cholesterol and mean levels of various lipoproteins revealed no correlations significantly greater than zero in study I and a correlation significantly greater than zero only in the case of Sf 12-20 molecules in studies II and III.

\section{DISCUSSION}

These investigations provide information about several kinds of variability for three classes of beta lipoproteins and of total cholesterol among individuals who were living in a controlled environment with no appreciable variation in activity and diet from day-to-day. Their failure to demonstrate significant differences in variability among various age groups implies that the homeostatic mechanisms for these serum lipids may not undergo alteration with age. While variability could not be related to age directly it is apparent that the characteristic increase of lipid levels with age to a maximum in the sixth decade $(13,19)$ would indirectly lead to some change in variability with age. Since subjects were selected for these studies to provide a similar range of Sf 12-20 levels in each age category, the usual age trends in level were not observed.

These studies indicate that technical error $\left(S_{E}\right)$ and biologic variability $\left(S_{B}\right)$ (and consequently over-all variability $\left(S_{T}\right)$ ) increase with the mean level among individuals. Inspection of available clinical information obtained during the ten-week period of these studies revealed no explanation for the origin of this biologic variability. Changes in body weight (trivial in this situation), changes in environmental temperature, intercurrent upper respiratory infections, and differences among indi-

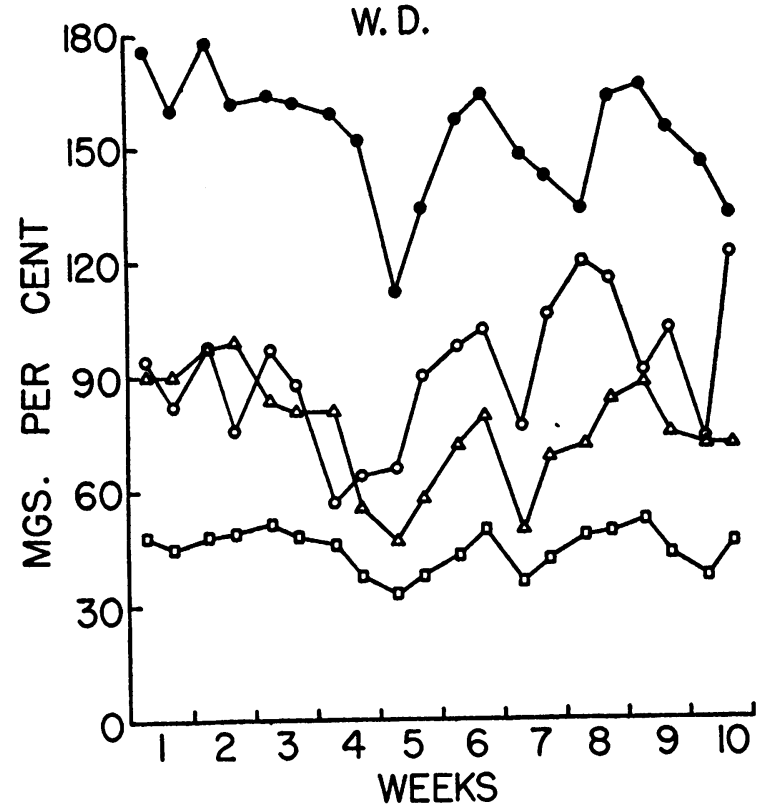

Fig. 1. Variations in Cholesterol and Certain Lipoprotein Levels Examined Twice Weekly for a Ten WeEk Period

Patient W. D., age 78. Triangles, Sf 12-20; squares, Sf 21-35; open circles, Sf 35-100; closed circles, total cholesterol.

viduals in physical activity were among the influences considered.

In addition to these net variations of serum lipid quantities within an individual over a 10 week period, another aspect of this variation deserves comment. This is the tendency of some individuals to show trends of change, either unidirectional or at times suggesting a periodic phenomenon. Such variations are obscured by the condensation of individuals' data into means and measures of range of single measurements. An example of this variation is shown in Figure 1. Attempts have been made to reduce these phenomena to some order by the application of one or another of the available statistical techniques for dealing with nonrandom changes. When tests for serial correlation (20) between successive bleedings were applied to the Sf 12-20 data of 46 subjects for whom complete sets of data for the 10-week study periods were available, there were five subjects with serial correlation coefficients significantly different from zero at the five per cent or less level of probability. This frequency (or a greater frequency) of significant coefficients is of 
small enough probability $(p=0.08)$ to suggest that there may be real trends in certain individuals. These are not now explicable but their existence must be recognized as indeed it must be in any body of serial biologic observations. The hazard which their presence lends to the interpretation of either diagnostic or therapeutic studies and for that matter any statistical analysis must be appreciated.

From a practical standpoint the data acquired in this investigation provide quantitative information on the magnitude, at any given serum level, of the overall variation, the technical error and the biological variability of the lipids studied. It is implicit in the regressions of $S_{T}, S_{E}$, and $S_{B}$ on mean lipoprotein levels that high levels are subject to greater fluctuation than low. From this it may be inferred that caution should be exercised in interpretations of diagnostic, prognostic or therapeutic importance based on values obtained from single or a limited number of blood samples from a given individual, especially when these values are elevated. With respect to total cholesterol, it is of interest that $S_{\mathrm{E}}$ increases with level but that $S_{T}$ and $S_{B}$ do not.

The data also permit estimation of the number of samples ( $\mathrm{n}$ ) required to yield an average level of any desired probability within any specified distance (d) of the true mean. This is accomplished by determining $\mathrm{n}$ from the relation
$\mathrm{n}=\frac{\mathrm{S}_{\mathrm{T}}{ }^{2} \mathrm{t}^{2}}{\mathrm{~d}^{2}}$, where $\mathrm{S}_{\mathrm{T}}$ is the standard deviation obtained from the regression of $S_{T}$ on $\bar{x}$ and $t$ is the standardized normal deviate exceeded in absolute value any desired percentage of the time. Sample sizes ( $n$ ) from a given individual required for a 67 per cent probability $\left(t_{.8 s}\right)$ that the average level will be within a distance (d) of the true mean are tabulated for three classes of lipoproteins and for total cholesterol in Table VII. Inspection of the $50 \mathrm{mg}$. per cent level for Sf 12-20 molecules indicates a single sample adequate to insure a value within ten mg. per cent of the true mean 67 per cent of the time. A sample size of three would insure a value within five $\mathrm{mg}$. per cent of the mean 67 per cent of the time. Inspection of the table for cholesterol, where $S_{T}$ is essentially the same for all levels, indicates a single sample adequate to insure a value within $15 \mathrm{mg}$. per cent of the true mean 67 per cent of the time. A sample size of two would insure a value within ten mg. per cent of the true mean 67 per cent of the time. These examples assume that the values for $S_{E}$ are comparable to those observed in this laboratory and that our estimate of $S_{B}$ is a reasonable approximation of the true standard deviation of distribution of the measure involved.

Levels of $50 \mathrm{mg}$. per cent for Sf 12-20 molecules and $250 \mathrm{mg}$. per cent for total cholesterol may be thought of as levels roughly separating the

TABLE VII

Number of samples (n) required for 67 per cent probability that the observed average level will fall within the distance $(d)$ of the true level

\begin{tabular}{|c|c|c|c|c|c|c|c|c|c|c|c|c|c|c|}
\hline \multirow{2}{*}{ Material } & \multirow{2}{*}{$\begin{array}{c}\overline{\mathbf{x}} \\
m \boldsymbol{g} .\end{array}$} & \multirow{2}{*}{$\begin{array}{c}\mathrm{ST} \\
\boldsymbol{m g} . \%\end{array}$} & \multicolumn{4}{|c|}{$\underset{m g .}{\mathrm{d}} \%$} & \multirow{2}{*}{ Material } & \multirow{2}{*}{$\begin{array}{c}\bar{x} \\
m \boldsymbol{m} . \%\end{array}$} & \multirow{2}{*}{$\underset{m g .}{\mathrm{ST}} \%$} & \multicolumn{5}{|c|}{ ms. $\%$} \\
\hline & & & 2.5 & 5 & 10 & 15 & & & & 2.5 & 5 & 10 & 15 & 20 \\
\hline$S_{1} 12-20$ & $\begin{array}{l}10 \\
20 \\
30 \\
40 \\
50 \\
60 \\
70 \\
80\end{array}$ & $\begin{array}{r}3.16 \\
4.32 \\
5.48 \\
6.64 \\
7.80 \\
8.96 \\
10.12 \\
11.28\end{array}$ & $\begin{array}{r}2 \\
3 \\
5 \\
8 \\
10 \\
13 \\
17 \\
21\end{array}$ & $\begin{array}{l}1 \\
1 \\
2 \\
2 \\
3 \\
4 \\
5 \\
6\end{array}$ & $\begin{array}{l}1 \\
1 \\
1 \\
1 \\
1 \\
1 \\
2 \\
2\end{array}$ & $\begin{array}{l}1 \\
1 \\
1 \\
1 \\
1 \\
1 \\
1 \\
1\end{array}$ & $S_{f} 35-100$ & $\begin{array}{r}10 \\
20 \\
30 \\
40 \\
50 \\
60 \\
80 \\
100\end{array}$ & $\begin{array}{r}4.82 \\
6.78 \\
8.74 \\
10.70 \\
12.66 \\
14.62 \\
18.54 \\
22.46\end{array}$ & $\begin{array}{r}4 \\
8 \\
13 \\
19 \\
26 \\
35 \\
55 \\
81\end{array}$ & $\begin{array}{r}1 \\
2 \\
4 \\
5 \\
7 \\
9 \\
14 \\
21\end{array}$ & $\begin{array}{l}1 \\
1 \\
1 \\
2 \\
2 \\
3 \\
4 \\
6\end{array}$ & $\begin{array}{l}1 \\
1 \\
1 \\
1 \\
1 \\
1 \\
2 \\
3\end{array}$ & $\begin{array}{l}1 \\
1 \\
1 \\
1 \\
1 \\
1 \\
1 \\
2\end{array}$ \\
\hline$S_{\&} 21-35$ & $\begin{array}{r}5 \\
10 \\
15 \\
20 \\
25 \\
30 \\
40 \\
50\end{array}$ & $\begin{array}{l}2.19 \\
2.90 \\
3.61 \\
4.32 \\
5.03 \\
5.74 \\
7.16 \\
8.58\end{array}$ & $\begin{array}{r}1 \\
2 \\
3 \\
3 \\
5 \\
6 \\
9 \\
12\end{array}$ & $\begin{array}{l}1 \\
1 \\
1 \\
1 \\
2 \\
2 \\
3 \\
3\end{array}$ & $\begin{array}{l}1 \\
1 \\
1 \\
1 \\
1 \\
1 \\
1 \\
1\end{array}$ & & $\begin{array}{l}\text { Total } \\
\text { Cholesterol }\end{array}$ & Any & 13 & 28 & 7 & 2 & 1 & 1 \\
\hline
\end{tabular}


normal from the abnormal $(14,19)$. This separation is most frequently attempted in the fourth, fifth, and sixth decades of age when the level and variability of the individual tested are near their maximum.

To obtain an indication of the accuracy with which a single measurement (Sf 12-20, cholesterol, etc.) can be placed on the continuum of measurements of individuals, an index formed from the ratio of $S_{T}$ to $S_{d}$ (the standard deviation of distribution of measurements of individuals) may be used. For an estimate of $S_{d}$, the standard deviation of distribution of measures for all 68 subjects has been used. Because of the selection of these individuals to maximize spread of the measures, $S_{d}$ is probably an overestimate of $\sigma_{d}$ (universe value), thus underestimating the ratio $\sigma_{\mathbf{T}} / \sigma_{\mathrm{d}}$. The value of the index of precision for Sf $12-20$ molecules at the $50 \mathrm{mg}$. per cent level is $.47(7.80 / 16.70)$; for cholesterol at the $250 \mathrm{mg}$. per cent level, $.32(13 / 40.25)$. This implies that a single sampling of Sf 12-20 will locate an individual within .47 of a $S_{d} 67$ per cent of the time; of cholesterol, within .32 of a $S_{d} 67$ per cent of the time. Thus cholesterol appears a slightly more accurate measure of "true" cholesterol level than Sf $12-20$ is of "true" Sf 12-20 level. From this the inference may be drawn that interpretations based on single samples of Sf 12-20 should be slightly more cautious than these based on a single sample of cholesterol.

There is no indication from these studies that a positive correlation exists between total cholesterol level and the levels of any or all of the lipoprotein molecules measured. This may be due to bias in the sampling (selection for level) or may reflect an inadequate sample size for showing significant correlation (especially if the correlation is low).

\section{SUMMARY}

The nature of the variability of the serum cholesterol and certain classes of beta lipoproteins has been studied in 68 adult males. The subjects were for the most part institutionalized for indigence not sickness and were living under known conditions of diet and activity. They were selected to represent a variety of ages and serum lipid levels.

These studies indicate the magnitude of overall variability, of technical error and of biologic variability for three classes of lipoproteins and for total cholesterol. They fail to demonstrate any change in variability related directly to the age of the subject but they do indicate a rise in technical error with mean level for all fractions, including cholesterol, and a linear relationship of the total and biologic variation to the mean level for lipoproteins. They provide data from which an estimate of sample size necessary to provide an average level of any desired probability within any specified distance of the true mean may be obtained. Certain limitations of single measurements of these serum quantities are pointed out, in particular, single measurements of Sf 12-20 lipoproteins at levels above $50 \mathrm{mg}$. per cent must be interpreted with caution.

\section{ACKNOWLEDGMENTS}

We appreciate the valuable suggestions of Drs. Nathan W. Shock, Milton Landowne, Felix Moore and Frederick J. Stare in the interpretation of data and the preparation of the manuscript.

We are grateful to Mrs. Elsie S. Beard for numerous computations; to Mr. Donald W. MacLean, ultracentrifuge engineer; and to Miss Rita M. O'Connell for film reading and computations.

\section{REFERENCES}

1. Bloor, W. R., Biochemistry of the Fatty Acids and Their Compounds, the Lipids, N. Y., Reinhold Publishing Corporation, 1943, American Chemical Society Monograph Series No. 93.

2. Bang, L., Utber Lipämie I. Biochem. Ztschr., 1918, 90, 383.

3. McClure, C. W., and Huntsinger, M. E., Studies in fat metabolism. I. The influence on blood lipids of single foodstuffs. J. Biol. Chem., 1928, 76, 1.

4. Bruger, M., and Somach, I., The diurnal variations of the cholesterol content of the blood. J. Biol. Chem., 1932, 97, 23.

5. Boyd, E. M., Diurnal variations in plasma lipids. J. Biol. Chem., 1935, 110, 61.

6. Turner, K. B., and Steiner, A., A long term study of the variation of serum cholesterol in man. J. Clin. Invest., 1939, 18, 45.

7. Steiner, A., and Domanski, B., Serum cholesterol levels in coronary arteriosclerosis. Arch. Int. Med., 1943, 71, 397.

8. Man, E. B., and Gildea, E. F., Variations in lipemia of normal subjects. J. Biol. Chem., 1937, 119, 769.

9. Man, E. B., and Peters, J. P., Variations of serum lipids with age. J. Lab. \& Clin. Med., 1953, 41, 738.

10. Sperry, W. M., The concentration of total cholesterol in the blood serum. J. Biol. Chem., 1937, 117, 391. 
11. Schube, P. G., Variations in the blood cholesterol of man over a time period. J. Lab. \& Clin. Med., 1936, 22, 280.

12. Gofman, J. W., Jones, H. B., Lindgren, F. T., Lyon, T. P., Elliott, H. A., and Strisower, B., Blood lipids and human atherosclerosis. Circulation, 1950, $2,161$.

13. Jones, H. B., Gofman, J. W., Lindgren, F. T., Lyon, T. P., Graham, D. M., Strisower, B., and Nichols, A. V., Lipoproteins in atherosclerosis. Am. J. Med., 1951, 11, 358.

14. Gofman, J. W., Jones, H. B., Lyon, T. P., Lindgren, F., Strisower, B., Colman, D., and Herring, V., Blood lipids and human atherosclerosis. Circulation, 1952, 5, 119.

15. Chandler, H. L., Lawry, E. A., Potee, K. A., and Mann, G. V., Spontaneous and induced variations in serum lipoproteins. Circulation, 1953, 8, 723.
16. Abell, L. L., Levy, B. B., Brodie, B. B., and Kendall, F. E., A simplified method for the estimation of total cholesterol in serum and demonstration of its specificity. J. Biol. Chem., 1952, 195, 357.

17. Gofman, J. W., Lindgren, F. T., Elliott, H. A., Mantz, W., Hewitt, J., Strisower, B., and Herring, $\mathrm{V}$., The role of lipids and lipoproteins in atherosclerosis. Science, 1950, 111, 166.

18. Grubbs, F. E., Sample criteria for testing outlying observations. Ann. Math. Stat., 1950, 21, 27.

19. Keys, A., Mickelsen, O., Miller, E. O., Hayes, E. R., and Todd, R. L., The concentration of cholesterol in the blood serum of normal man and its relation to age. J. Clin. Invest., 1950, 29, 1347.

20. Dixon, W. J., Further contributions to the problem of serial correlation. Ann. Math. Stat., 1944, 15, 119. 